\title{
Analytical Investigation of Peristaltic Transport of Power Law Fluid through a Tube
}

\author{
K Sadeghi and H Jalali Talab*
}

School of Mechanical Engineering, College of Engineering, University of Tehran, Tehran, Iran

\begin{abstract}
In this paper, the peristaltic flow of a power law fluid in a cylindrical channel is studied analytically. The governing equations consist of continuity and momentum equations are solved mathematically by perturbation method. The zeroth and first order in amplitude ratio of the stream function is considered for perturbation series. The effects of flow behavior index $(n)$ flow rate and amplitude ratio $(\varepsilon)$ on the flow field are investigated. The results show that increment of power law index predicts more flow rate and less pressure rise in peristaltic motion. Trapping phenomenon is form by closed stream line depending on peristaltic motion.
\end{abstract}

Keywords: Peristaltic motion; Power law fluid; Perturbation method; Cylindrical cannel

\section{Introduction}

Analysis of peristaltic flow has great practical importance in many biological and biomedical systems such as the flow of urine through the urethra, the swallowing process through the esophagus, the movement of spermatozoa in the ducts efferent of the male reproductive tract, transport of lymph in the lymphatic vessels and in the vasomotor of small blood vessels such as arterioles, venules and capillaries. Latham [1] was probably the first to investigate the mechanism of peristalsis in relation to mechanical pumping. Primary analytical studies of peristaltic transport for Newtonian fluids were performed by Shapiro et al. [2] in wave frame and by Fung and Yih [3] in laboratory frame. Peristaltic flow of a power-law fluid in a channel under long wavelength approximation was studied by Radhakrishnamacharya [4]. Srivastava and Srivastava [5] were investigated the peristaltic flow in blood vessels for the Casson fluid. Mernone and Mazumdar studied Casson fluid in Peristaltic motion by perturbation method [6]. The peristaltic transport of a power-law fluid in the male reproductive tract was studied by Srivastava and Srivastava [7]. Peristaltic pumping of two layered powerlaw fluids in cylindrical tube was investigated by Usha and Rao [8]. Eytan and Elad [9] and Eytan et al. [10] have studied the intra uterine fluid flow in the sagittal cross section of the uterus by an asymmetric channel under lubrication approach. The lubrication approach assumes that the width of the channel is very small compared with length. Mishra and Rao [11] have studied the peristaltic flow in asymmetric channels with asymmetry generated by different amplitudes of the peristaltic waves in addition to different phases. Takabatake and Ayukava [12] and Takabatake et al. [13] studied the problem of urine flow through a channel and a circular cylindrical tube, respectively by employing finite difference technique and discussed the phenomena of trapping and reflux. Siddiqui and Schwars studied on trapping for second-order fluid [14]. Recent investigations focused on numerical simulation of peristaltic flow in large variety of geometries [15-21].

In this paper, the peristaltic flow of a power law fluid in a cylindrical channel is investigated by using a perturbation method in the wave frame. The effects of main parameters of flow rate, Reynolds number, flow behavior index $(n)$ and amplitude ratio $(\varepsilon)$ on the flow regime are studied.

\section{Governing equations}

The physical model and related parameters are illustrated in the
Figure 1. The dimensionless parameters are defined as follows:

$$
\begin{aligned}
& \varepsilon=\frac{A}{d} \bar{Z}=\frac{Z}{d} \bar{R}=\frac{R}{d} \bar{G}=\frac{G}{d} \bar{t}=\frac{c t}{d} \\
& \alpha=\frac{2 \pi d}{\lambda} \bar{v}_{R}=\frac{v_{R}}{c} \bar{\nu}_{Z}=\frac{\nu_{Z}}{c} \bar{\psi}=\frac{\psi}{c d^{2}} \quad \bar{Q}=\frac{Q}{c d^{2}} \quad \bar{p}=\frac{p}{\rho d^{2}} \operatorname{Re}=\frac{d^{n} c^{2-n} \rho}{m}
\end{aligned}
$$

It is imagined that $\alpha^{2}$ is in the order of $\varepsilon$ and the wave equation propagated along the tube is cosine form:

$$
G(Z, t)=d+A \cos \frac{2 \pi d}{\lambda}(Z-c t)
$$

In the above equation $d$ is the half non-deformed width of the channel, $A$ is wave amplitude, $\lambda$ is wave length, $Z$ is longitudinal coordinate along the tube, $c$ is wave velocity and $t$ is time. Peristaltic motion is an unsteady phenomenon instinctively but it can be assumed steady if the coordinates travels with the velocity of wave so as it is shown in Figure 1, two fixed (laboratory frame) and moving (wave frame) coordinate can be defined in the following relation:

$$
\mathrm{Z}=\mathrm{z}+\mathrm{ct}, \quad \mathrm{R}=\mathrm{r}
$$

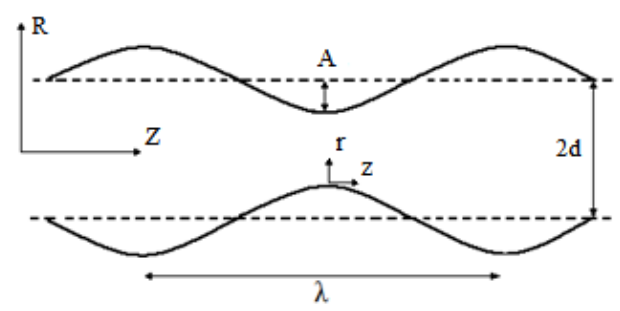

Figure 1: Illustration of peristaltic motion.

*Corresponding author: $\mathrm{H}$ Jalali Talab, School of Mechanical Engineering, College of Engineering, University of Tehran, Tehran, Iran, Tel: 00989126831475; E-mail: h.jalalitalab@gmail.com

Received December 16, 2013; Accepted January 07, 2014; Published January 15,2014

Citation: Sadeghi K, Jalali Talab H (2014) Analytical Investigation of Peristaltic Transport of Power Law Fluid through a Tube. J Appl Mech Eng 3: 136. doi:10.4172/2168-9873.1000136

Copyright: @ 2014 Sadeghi K, et al. This is an open-access article distributed under the terms of the Creative Commons Attribution License, which permits unrestricted use, distribution, and reproduction in any medium, provided the original author and source are credited. 


$$
\mathcal{V}_{Z}=\mathcal{V}_{z}+c \quad v_{R}=v_{r} \text {, }
$$

Where $\left(v_{Z}, v_{z}\right)$ and $\left(v_{R}, v_{r}\right)$ are the velocity component in the laboratory and wave frame for axial and radial direction respectively. $\mathrm{Z}$-axis is the center line and $\mathrm{R}$ is the distance measured radial.

Governing equations consist of continuity and momentum equations in laboratory frame are in the following form:

\section{Continuity}

$$
\frac{\partial \rho}{\partial t}+\frac{1}{R} \frac{\partial}{\partial R}\left(\rho R v_{R}\right)+\frac{1}{R} \frac{\partial}{\partial \theta}\left(\rho v_{\theta}\right)+\frac{\partial}{\partial Z}\left(\rho v_{Z}\right)=0
$$

\section{R-Momentum}

$$
\begin{aligned}
& \rho\left(\frac{\partial v_{R}}{\partial t}+v_{R} \frac{\partial v_{R}}{\partial R}+\frac{v_{\theta}}{R} \frac{\partial v_{R}}{\partial \theta} \frac{v_{\theta}^{2}}{R}+v_{z} \frac{\partial v_{R}}{\partial Z}\right)= \\
& -\frac{\partial \rho}{\partial R}+\left[\frac{1}{R} \frac{\partial}{\partial R}\left(R_{\tau R R}\right)+\frac{1}{R} \frac{\partial}{\partial \theta}(\tau \theta R)+\frac{\partial}{\partial Z}(\tau Z R) \frac{\tau_{\theta \theta}}{R}\right]
\end{aligned}
$$

\section{Z-Momentum}

$$
\begin{aligned}
& \rho\left(\frac{\partial v_{Z}}{\partial t}+v_{R} \frac{\partial v_{Z}}{\partial R}+\frac{v_{\theta}}{R} \frac{\partial v_{Z}}{\partial \theta}+v_{z} \frac{\partial v_{Z}}{\partial Z}\right)= \\
& -\frac{\partial \rho}{\partial Z}+\left[\frac{1}{R} \frac{\partial}{\partial R}\left(R_{\tau R Z}\right)+\frac{1}{R} \frac{\partial}{\partial \theta}(\tau \theta Z)+\frac{\partial}{\partial Z}(\tau Z Z)\right]
\end{aligned}
$$

Also the boundary conditions are:

$$
\begin{aligned}
& v_{Z}=0, v_{R}=\frac{\partial}{\partial t} G(Z, t)=-c \frac{\partial G}{\partial Z} @ R=G(Z, t) \\
& v_{R}=0, \frac{\partial v_{Z}}{\partial R}=0 @ R=0
\end{aligned}
$$

In the above equations for obtaining the stress term for power law fluid, (power law fluid is considered as a simple shear fluid) the constructive equation is defined as:

$$
\eta\left(I I_{2 D}\right)=m \gamma^{.(n-1)} \tau_{i, j}=\eta \gamma_{i j}=m \gamma_{i j}^{. n}
$$

Where $I I_{2 D}$ is second invariant of strain rate tensor, $n$ is flow behavior index, $m$ is flow consistency and $\gamma_{i j}$ is defined as shear rate:

$$
\gamma_{i j}=2 D=\left(\frac{\partial \boldsymbol{u}_{i}}{\partial \boldsymbol{x}_{j}}+\frac{\partial \boldsymbol{u}_{j}}{\partial \boldsymbol{x}_{i}}\right)
$$

Let $u_{i}$ and $u_{j}$ be the longitudinal $v_{R}$ and transverse $v_{z}$ velocity components respectively, the stress tensor will be:

$$
\tau_{i j}=\left[\begin{array}{ccc}
m\left(2 \frac{\partial \mathcal{V}_{R}}{\partial R}\right)^{n} & 0 & m\left(\frac{\partial \mathcal{V}_{R}}{\partial Z}+\frac{\partial \mathcal{V}_{Z}}{\partial R}\right)^{n} \\
0 & m\left(2 \frac{\mathcal{V}_{R}}{R}\right)^{n} & 0 \\
m\left(\frac{\partial \mathcal{V}_{R}}{\partial Z}+\frac{\partial \mathcal{V}_{Z}}{\partial R}\right)^{n} & 0 & m\left(2 \frac{\partial \mathcal{V}_{R}}{\partial R}\right)^{n}
\end{array}\right]
$$

By defining stream function for $2 D$ flow and applying dimensionless parameters the non-dimensional governing equations and boundary conditions become as follows:

$$
v_{R}=-\frac{1}{R} \frac{\partial \psi}{\partial Z} \quad v_{Z}=\frac{1}{R} \frac{\partial \psi}{\partial R}
$$

\section{$\bar{R}$-Momentum}

$\operatorname{Re}\left[-\frac{1}{\bar{R}} \bar{\psi}_{\overline{\bar{z}} \bar{t}}+\frac{1}{\bar{R}} \bar{\psi}_{\overline{\bar{Z}}}\left(-\frac{1}{\bar{R}^{2}} \bar{\psi}_{\overline{\bar{Z}}}+\frac{1}{\bar{R}} \bar{\psi}_{\overline{\bar{R}} \bar{Z}}\right)-\frac{1}{\bar{R}^{2}} \bar{\psi}_{\bar{R}} \bar{\psi}_{\overline{\bar{Z}} \overline{\bar{Z}}}\right]=$

$-\operatorname{Re} \frac{\partial \bar{p}}{\partial \bar{R}}+m \frac{2^{n}}{R}\left(\frac{1}{\bar{R}^{2}} \bar{\psi}_{\overline{\bar{Z}}}-\frac{1}{\bar{R}} \bar{\psi}_{\bar{R} \bar{Z}}\right)^{n}$

$+m \cdot \bar{R} \cdot n \cdot 2^{n}\left(-\frac{2}{\bar{R}^{3}} \bar{\psi}_{\bar{Z}}+\frac{2}{\bar{R}^{2}} \bar{\psi}_{\overline{\bar{Z}} \overline{\bar{R}}}-\frac{1}{\bar{R}} \bar{\psi}_{\overline{\bar{Z} \bar{R} \bar{R}}}\right)\left({\overline{\bar{T}^{2}}}^{2} \bar{\psi}_{\overline{\bar{Z}}}-\frac{1}{\bar{R}} \bar{\psi}_{\bar{R} \bar{Z}}\right)^{n-1}$

$+m \cdot n\left(-\frac{1}{\bar{R}} \bar{\psi}_{\overline{\bar{Z} \bar{Z} \bar{Z}}}+\frac{1}{\bar{R}} \bar{\psi}_{\overline{\bar{Z} \bar{R} \bar{R}}}-\frac{1}{\bar{R}^{2}} \bar{\psi}_{\overline{\bar{Z} \bar{R}}}\right) \times$

$\left(-\frac{1}{\bar{R}} \bar{\psi}_{\bar{Z} \bar{Z}}+\frac{1}{\bar{R}} \bar{\psi}_{\bar{R} \bar{R}}-\frac{1}{\bar{R}^{2}} \bar{\psi}_{\bar{R}}\right)^{n-1}-m \frac{2^{n}}{\bar{R}}\left(-\frac{1}{\bar{R}^{2}} \bar{\psi}_{\bar{Z}}\right)^{n}$

\section{$\bar{Z}$-Momentum}

$\operatorname{Re}\left[\frac{1}{\bar{R}} \bar{\psi}_{\bar{R} \bar{t}}-\frac{1}{\bar{R}} \bar{\psi}_{\bar{Z}}\left(-\frac{1}{\bar{R}^{2}} \bar{\psi}_{\bar{R}}+\frac{1}{\bar{R}} \bar{\psi}_{\bar{R} \bar{R}}\right)+\frac{1}{\bar{R}^{2}} \bar{\psi}_{\bar{R}} \bar{\psi}_{\bar{R} \bar{Z}}\right]=$

$-\operatorname{Re} \frac{\partial \bar{p}}{\partial \bar{Z}}+m \frac{1}{\bar{R}}\left(-\frac{1}{\bar{R}} \bar{\psi}_{\bar{Z} \bar{Z}}+\frac{1}{\bar{R}} \bar{\psi}_{\bar{R} \bar{R}}-\frac{1}{\bar{R}^{2}} \bar{\psi}_{\bar{R}}\right)^{n}$

$+m \cdot n\left(\frac{1}{\bar{R}^{2}} \bar{\psi}_{\bar{Z} \bar{Z}}-\frac{1}{\bar{R}} \bar{\psi}_{\overline{\bar{Z}} \overline{\bar{R}} \bar{Z}}+\frac{2}{\bar{R}^{3}} \bar{\psi}_{\overline{\bar{R}}}-\frac{2}{\bar{R}^{2}} \bar{\psi}_{\overline{\bar{R}} \bar{R}}+\frac{1}{\bar{R}} \bar{\psi}_{\overline{\bar{R}} \bar{R} \bar{R}}\right) \times$

$\left(-\frac{1}{\bar{R}} \bar{\psi}_{\overline{Z \bar{Z}}}+\frac{1}{\bar{R}} \bar{\psi}_{\bar{R} \bar{R}}-\frac{1}{\bar{R}^{2}} \bar{\psi}_{\bar{R}}\right)^{n-1}$

$+m \cdot n \cdot 2^{n}\left(+\frac{1}{\bar{R}} \bar{\psi}_{\bar{R} \bar{Z} \bar{Z}}\right)\left(+\frac{1}{\bar{R}} \bar{\psi}_{\bar{R} \bar{Z}}\right)^{n-1}$

And non-dimensional boundary conditions:

$\bar{\psi}_{\bar{R}} \approx \bar{\psi}_{0 \bar{R}}+\varepsilon \bar{\psi}_{1 \bar{R}}=0 @ \bar{R}=\bar{G}(Z, t)$

$-\frac{1}{\bar{R}} \bar{\psi}_{\bar{Z}} \approx-\frac{1}{\bar{R}}\left(\bar{\psi}_{0 \bar{Z}}+\varepsilon \bar{\psi}_{\overline{1} \bar{Z}}\right)=-\frac{\partial \bar{G}}{\partial \bar{Z}}=\varepsilon \alpha \sin \alpha(\bar{Z}-\bar{t})$

@ $\bar{R}=\bar{G}(Z, t)$

$\left(\frac{1}{\bar{R}^{2}} \bar{\psi}_{0 \bar{R}}+\frac{1}{\bar{R}} \bar{\psi}_{0 \bar{R} \bar{R}}\right)+\varepsilon\left(\frac{1}{\bar{R}^{2}} \bar{\psi}_{1 \bar{R}}+\frac{1}{\bar{R}} \bar{\psi}_{1 \bar{R} \bar{R}}\right)=0 @ \bar{R}=0$

$\bar{\psi}_{\overline{\bar{z}}} \approx \bar{\psi}_{0 \overline{\bar{Z}}}+\varepsilon \bar{\psi}_{1 \overline{\bar{Z}}}=0 @ \bar{R}=0$

Solution Procedure (Zeroth-Order Approximation): By expressing stream function $\psi$ as a series in terms of amplitude ratio $\varepsilon$ we have

$$
\psi=\psi_{0}+\varepsilon \psi_{1}+o\left(\varepsilon^{2}\right)
$$

The axial pressure gradient is assumed to be zeroth-order and linear. Therefore the term is a function of $\mathrm{R}$ only through the domain. 


$$
p=p_{0} \psi=\psi_{0}(R)
$$

By substituting the stream function series in momentum equations for the zeroth-order momentum equations will be:

\section{$\bar{R}$-Momentum:}

$\operatorname{Re} \frac{\partial \overline{p_{0}}}{\partial \bar{R}}=0$

\section{$\bar{Z}$-Momentum:}

$\operatorname{Re} \frac{\partial \bar{p}_{0}}{\partial \bar{R}}=\frac{1}{\bar{R}}\left(\frac{1}{\bar{R}} \bar{\psi}_{0 \bar{R} \bar{R}}-\frac{1}{\bar{R}^{2}} \bar{\psi}_{0 \bar{R}}\right)^{n}+$

.$n\left({\frac{2}{\bar{R}^{3}}}^{3} \bar{\psi}_{0 \bar{R}}-\frac{2}{\bar{R}^{2}} \bar{\psi}_{0 \bar{R} \bar{R}}+\frac{1}{\bar{R}} \bar{\psi}_{0 \bar{R} \bar{R} \bar{R}}\right)\left(\frac{1}{\bar{R}} \bar{\psi}_{0 \bar{R} \bar{R}}-\frac{1}{\bar{R}^{2}} \bar{\psi}_{0 \bar{R}}\right)^{n-1}$

The $\bar{Z}$-momentum equations with the boundary conditions (13a, 13c) can be calculated analytically and $\psi_{0}$ and $\Delta p$ will be:

$\bar{\psi}_{0}=B \frac{n}{n+1}\left(-\frac{1}{2} \bar{R}^{2}+\frac{n}{3 n+1} \bar{R}^{\left(3+\frac{1}{n}\right)}\right)$

Where:

$B=\left(\frac{\operatorname{Re}}{2} \frac{\partial \bar{p}_{0}}{\partial \bar{Z}}\right)^{\frac{1}{n}}$

The flow rate is found by the following relation:

$\bar{q}=\int_{0}^{2 \pi} \int_{0}^{1+\bar{G}} \bar{R} \cdot v_{\bar{Z}} \cdot d \bar{R} \cdot d \theta$

As we had:

$\bar{\nu}_{\bar{Z}}=\frac{1}{\bar{R}} \frac{\partial \bar{\psi}}{\bar{R}}$

So

$\bar{q}=2 \pi(\bar{\psi}(1+\varepsilon \cos \alpha(\bar{Z}-\bar{t}))-\bar{\psi}(0))$

Taylor expansion results:

$$
\bar{q} \approx 2 \pi\left(\bar{\psi}(1)+\varepsilon \cos \alpha(\bar{Z}-\bar{t}) \bar{\psi}_{\bar{R}}(1)\right)
$$

It is important to find the flow over a period of time $\bar{T}$ at the fixed cross section position that is called time-mean flow as:

$\bar{Q}=\frac{1}{\bar{T}} \int_{0}^{\bar{T}} \bar{q} d \bar{t}$

Where $\bar{T}$ is:

$\bar{T}=\frac{\lambda}{c}$

Finally:

$\bar{Q}=\frac{2 \pi}{\bar{T}} \int_{0}^{\bar{T}} B\left(\frac{-n}{2(3 n+1)}\right) d \bar{t}+\frac{2 \pi}{\bar{T}} \int_{0}^{\bar{T}} \varepsilon \cos \alpha(\bar{Z}-\bar{t}) d \bar{t}$

Since the second part of right hand side of above equation is zero the pressure gradient is considered as:

$$
\frac{d \bar{p}}{d \bar{Z}}=\frac{2}{\operatorname{Re}}\left(\frac{-Q}{2 \pi(n / 2)(3 n+1)}\right)^{n}
$$

The non-dimension pressure rise over a wave length is defined in the following form:

$$
\Delta \bar{p}=\int_{0}^{\lambda / d} \frac{d \bar{p}}{d \bar{Z}} d \bar{Z}
$$

And a relation is found for pressure rise in term of time-mean flow:

$$
\Delta \bar{p}_{i(\lambda / d)}=\frac{2}{\operatorname{Re}} \frac{\lambda}{d}\left[\frac{-\bar{Q}}{2 \pi(n / 2(3 n+1))}\right]^{n}
$$

Solution Procedure (First-Order Approximation): Assuming that there is no horizontal displacement of the tube walls during the peristaltic motion, by expanding the equations $(13 a, 13 b)$ about $R=1$ vertically, we have:

$$
\begin{aligned}
& \bar{\psi}_{\bar{R}}(1+\varepsilon \cos \alpha(\bar{Z}-\bar{t}))=\bar{\psi}_{\bar{R}}(1)+\delta \bar{\psi}_{\bar{R} \bar{R}}(1)+\frac{\delta^{2}}{2} \bar{\psi}_{\bar{R} \bar{R} \bar{R}}(1) \\
& +\frac{\delta^{3}}{6} \bar{\psi}_{\bar{R} \bar{R} \bar{R} \bar{R}}(1)+o\left(\delta^{4}\right)=0 \\
& -\frac{1}{1+\delta} \bar{\psi}_{\bar{Z}}(1+\delta)=-\left(1-\delta+\delta^{2}-o\left(\delta^{3}\right)\right) \times \\
& \left(\bar{\psi}_{\bar{Z}}(1)+\delta \bar{\psi}_{\bar{Z} \bar{R}}(1)+\frac{\delta^{2}}{2} \bar{\psi}_{\bar{Z} \bar{R} \bar{R}}(1)+o\left(\delta^{3}\right)\right)=\varepsilon \alpha \cos \alpha(\bar{Z}-\bar{t})
\end{aligned}
$$

Where:

$$
\delta=\varepsilon \cos \alpha(\bar{Z}-\bar{t})
$$

And the results are:

$$
\begin{aligned}
& \bar{\psi}_{0 \bar{R}}(1) \approx 0 \\
& \bar{\psi}_{1 \bar{R}}(1) \approx-\bar{\psi}_{0 \bar{R} \bar{R}}(1) \cos \alpha(\bar{Z}-\bar{t}) \\
& \bar{\psi}_{1 \bar{R} \bar{R}}(1) \approx-\frac{1}{2} \bar{\psi}_{0 \bar{R} \bar{R} \bar{R}}(1) \cos \alpha(\bar{Z}-\bar{t}) \\
& \bar{\psi}_{1 \bar{R} \bar{R} \bar{R}}(1) \approx-\frac{1}{3} \bar{\psi}_{0 \bar{R} \bar{R} \bar{R}}(1) \cos \alpha(\bar{Z}-\bar{t}) \\
& \bar{\psi}_{1 \bar{Z}}(1) \approx-\alpha \sin \alpha(\bar{Z}-\bar{t})
\end{aligned}
$$

These boundary conditions suggest that the solution for $\bar{\psi}_{1}$ can obtained in the following form:

$$
\bar{\psi}_{1}(\bar{Z}, \bar{R}, \bar{t})=h(\bar{R}) \cos \alpha(\bar{Z}-\bar{t})+g(\bar{R}) \sin \alpha(\bar{Z}-\bar{t})
$$

Eliminating the pressure gradient using cross-differentiation and subtraction of momentum equations then substituting $\bar{\psi}_{0}$ and $\bar{\psi}_{1}$ from equations $(18,32)$, afterward neglecting the coefficients of $\varepsilon^{2}$ and $\varepsilon \alpha^{2}$, we will have: 
$\operatorname{Re}\left\{-h^{\prime} \alpha \sin \alpha(\bar{Z}-\bar{t})+g^{\prime} \alpha \cos \alpha(\bar{Z}-\bar{t})\right\}+$

$\operatorname{Re}\left\{\bar{R} h^{\prime \prime} \alpha \sin \alpha(\bar{Z}-\bar{t})-\bar{R} g{ }^{\prime \prime} \alpha \cos \alpha(\bar{Z}-\bar{t})\right\}+$

$\operatorname{Re} \frac{n}{n+1} B\left\{-h^{\prime} \alpha \sin \alpha(\bar{Z}-\bar{t})+g^{\prime} \alpha \cos \alpha(\bar{Z}-\bar{t})\right\}+$

$\operatorname{Re} \frac{n}{n+1} B\left(\bar{R}^{(2+1 / n)}-\bar{R}\right) \times$

$\left\{-h^{\prime \prime} \alpha \sin \alpha(\bar{Z}-\bar{t})+g^{\prime \prime} \alpha \cos \alpha(\bar{Z}-\bar{t})\right\} \times B^{(1-n)} \bar{R}^{(1 / n-1)}$

$=-\left(\frac{-1+2 n}{n^{2}}\right)\left\{\frac{h^{\prime \prime}}{\bar{R}} \cos \alpha(\bar{Z}-\bar{t})+\frac{g^{\prime \prime}}{\bar{R}} \sin \alpha(\bar{Z}-\bar{t})\right\}$

$-\left(\frac{1-2 n}{n^{2}}\right)\left\{\frac{h^{\prime}}{\bar{R}^{2}} \cos \alpha(\bar{Z}-\bar{t})+\frac{g^{\prime}}{\bar{R}^{2}} \sin \alpha(\bar{Z}-\bar{t})\right\}$

$+\frac{2(n-1)}{n}\left\{\frac{2 h^{\prime}}{\bar{R}^{2}} \cos \alpha(\bar{Z}-\bar{t})+\frac{2 g^{\prime}}{\bar{R}^{2}} \sin \alpha(\bar{Z}-\bar{t})\right\}$

$-\frac{2(n-1)}{n}\left\{\frac{2 h^{\prime \prime}}{\bar{R}} \cos \alpha(\bar{Z}-\bar{t})+\frac{2 g^{\prime \prime}}{\bar{R}} \sin \alpha(\bar{Z}-\bar{t})\right\}$

$+\frac{2(n-1)}{n}\left\{h^{\prime \prime \prime} \cos \alpha(\bar{Z}-\bar{t})+g^{\prime \prime \prime} \sin \alpha(\bar{Z}-\bar{t})\right\}$

$+\left\{\frac{4 h^{\prime \prime}}{\bar{R}} \cos \alpha(\bar{Z}-\bar{t})+\frac{4 g^{\prime \prime}}{\bar{R}} \sin \alpha(\bar{Z}-\bar{t})\right\}$

$+\left\{\bar{R} h^{\prime \prime \prime} \cos \alpha(\bar{Z}-\bar{t})+\bar{R} g^{\prime \prime \prime} \sin \alpha(\bar{Z}-\bar{t})\right\}$

$+\left\{\frac{4 h^{\prime}}{\bar{R}^{2}} \cos \alpha(\bar{Z}-\bar{t})+\frac{4 g^{\prime}}{\bar{R}^{2}} \sin \alpha(\bar{Z}-\bar{t})\right\}$

$-\left\{2 h^{\prime \prime \prime} \cos \alpha(\bar{Z}-\bar{t})+2 g^{\prime \prime \prime} \sin \alpha(\bar{Z}-\bar{t})\right\}$

By collecting the coefficients of sin and cosine in either side, two coupled equations are concluded as follows:

$$
\begin{aligned}
& \operatorname{Re}\left[g^{\prime} \alpha+\frac{n}{n+1} B g^{\prime} \alpha+\bar{R} g^{\prime \prime} \alpha+\frac{n}{n+1} B g^{\prime \prime} \alpha\left(\bar{R}^{\left(2+\frac{1}{n}\right)}-\bar{R}\right)\right] \\
& =B^{(n-1)} \frac{\left(1-\frac{1}{n}\right)}{} \times \\
& {\left[\left(\frac{-2 n+1}{n^{2}}\right)\left(\frac{h^{\prime \prime}}{\bar{R}}-\frac{h^{\prime}}{\bar{R}^{2}}\right)+\frac{2(n-1)}{n}\left(-\frac{2 h^{\prime \prime}}{\bar{R}}-\frac{2 h^{\prime}}{\bar{R}^{2}}+h^{\prime \prime \prime}\right)\right]} \\
& B^{(n-1)} \bar{R}^{\left(1-\frac{1}{n}\right)} \times\left(-\frac{4 h^{\prime \prime}}{\bar{R}}-\frac{4 h^{\prime}}{\bar{R}^{2}}-2 h^{\prime \prime \prime}+\bar{R} h^{\prime \prime \prime}\right)
\end{aligned}
$$

And

$\operatorname{Re}\left[-h^{\prime} \alpha-\frac{n}{n+1} B h^{\prime} \alpha+\bar{R} h^{\prime \prime} \alpha-\frac{n}{n+1} B h^{\prime \prime} \alpha\left(\bar{R}^{\left(2+\frac{1}{n}\right)}-\bar{R}\right)\right]$

$=B^{(n-1)} \frac{{ }^{\left(1-\frac{1}{n}\right)} \times}{}$

$\left[\left(\frac{-2 n+1}{n^{2}}\right)\left(\frac{g^{\prime \prime}}{\bar{R}}-\frac{g^{\prime}}{\bar{R}^{2}}\right)+\frac{2(n-1)}{n}\left(-\frac{2 g^{\prime \prime}}{\bar{R}}+\frac{2 g^{\prime}}{\bar{R}^{2}}+g^{\prime \prime \prime}\right)\right]$

$B^{(n-1)} \bar{R}^{\left(1-\frac{1}{n}\right)} \times\left(\frac{4 g^{\prime \prime}}{\bar{R}}-\frac{4 g^{\prime}}{\bar{R}^{2}}-2 g^{\prime \prime \prime}+\bar{R} g^{\prime \prime \prime}\right)$

With following boundary conditions based from equations $(13 a-13 d)$

$$
\begin{aligned}
& g^{\prime}(0)=0 \quad h^{\prime}(0)=0 \quad g(0)=0 \quad h(0)=0 \\
& g^{\prime}(1)=0 \quad h^{\prime}(1)=-B \quad g(1)=0 \quad h(1)=1
\end{aligned}
$$

The set of coupled ODE equations $(34,35)$ are very complicated therefore the numerical spectral method based on Chebychev series is applied to obtain $\mathrm{h}, \mathrm{g}$ and higher-order derivation of them.

\section{Result and Discussion}

The peristaltic motion of a power law fluid in a tube with a cosine wave propagating along the boundary has been considered under the assumption that the wave amplitude is small compared to half nondeformed diameter of flexible tube. The stream function and the velocity components are obtained as power series, in terms of the amplitude of deformation divided by tube diameter. The Reynolds number is arbitrary but the flow remains laminar. It is imagined that the pressure changes linearly along the tube and it is uniform in each cross-section.

Figure 2 shows the variations of pressure difference versus timemean flow rate over the wave period. As it is shown in Figure 2 the pressure rise decreases by increasing flow rate and the maximum flow rate is related to zero pressure rise. For Newtonian case $(n=1)$ the diagram is linear as indicated by Mernone and Mazumdar [6]. Shear thinning fluid $(n<1)$ diagrams are under the Newtonian fluid diagram with downward arc shaped as shown by Sirvastava and Sirvastava [7] but shear thickening fluid diagrams maintain on top of it with upward arc shaped. The diagrams show that in cases $(n>1)$ the flow variation has small effect on pressure rise near to zero and it is vice versa for cases

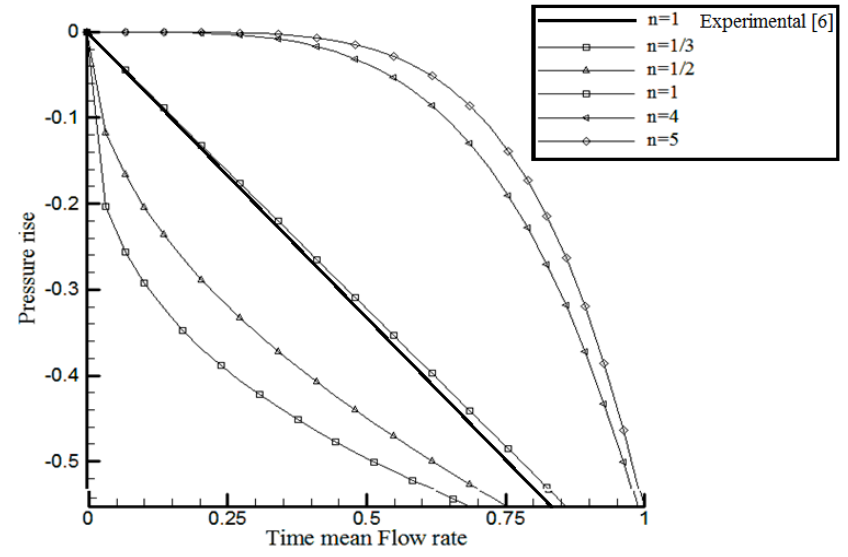

Figure 2: Variations of pressure rise versus flow rate and $(\operatorname{Re}=50, \varepsilon=0.05$, $\alpha=0.5)$.

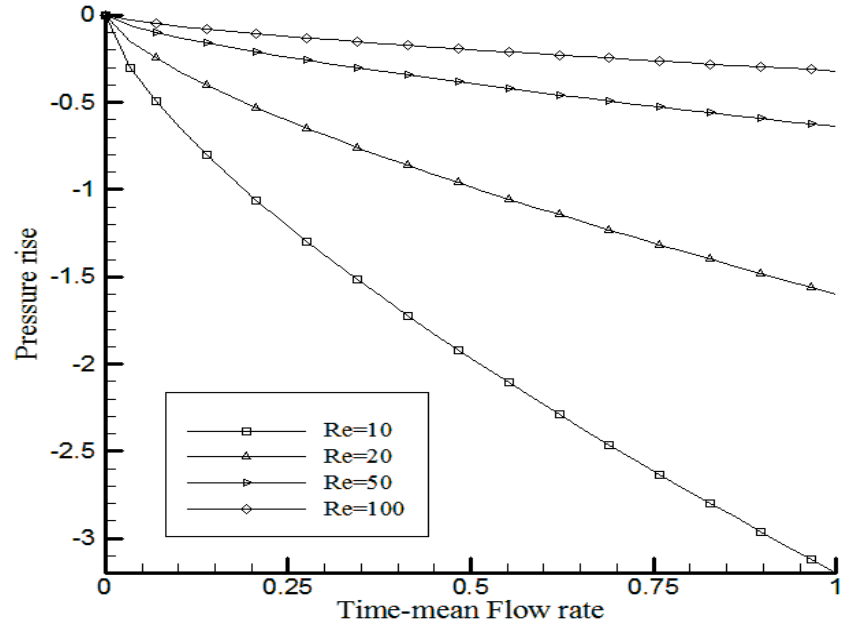

Figure 3: Variations of pressure rise versus flow rate of a shear thinning fluid for different Reynolds and $(n=0.7, \varepsilon=0.05, \alpha=0.5)$. 


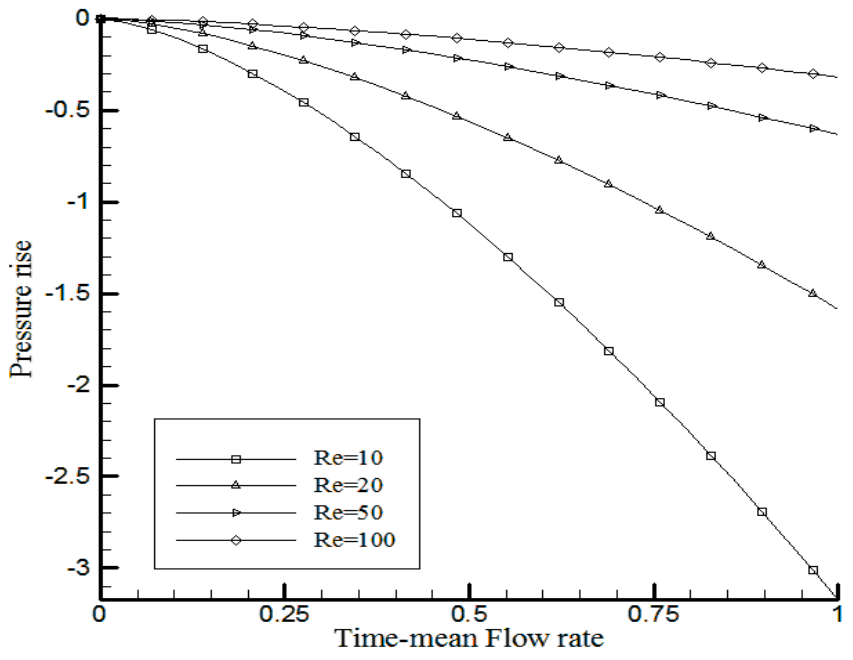

Figure 4: Variations of pressure rise versus flow rate of a shear thickening fluid for different Reynolds and $(n=1.5, \varepsilon=0.05, \alpha=0.5)$.

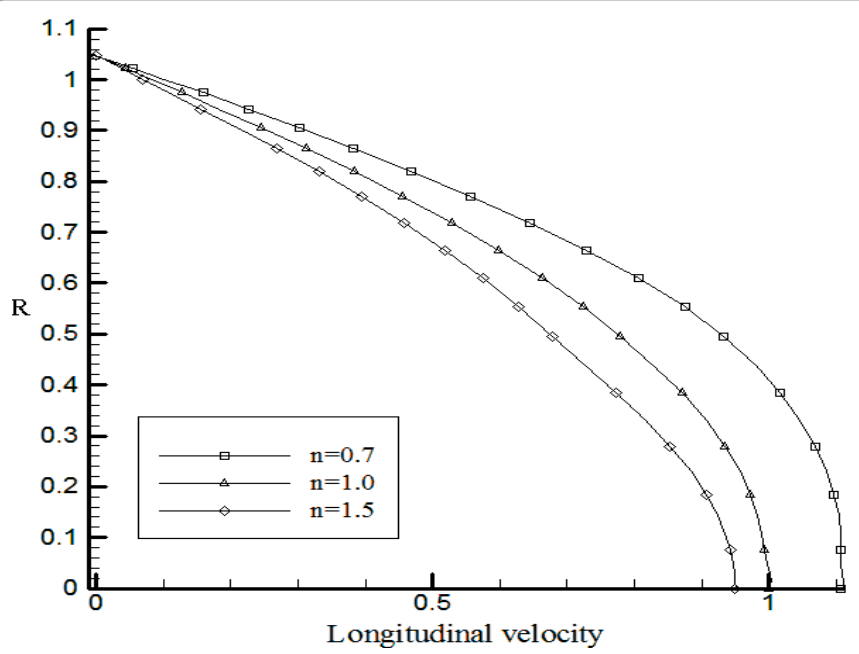

Figure 5: Axial velocity profile for a fixed position, different power law index and $(\varepsilon=0.05, \alpha=0.5, \operatorname{Re}=50)$.

$(\mathrm{n}<1)$. In both shear thinning and shear thickening fluid increasing power law index brings more pressure rise for the fixed time-mean flow rate.

Figures 3 and 4 show the pressure rise in term of time-mean flow rate for two different power law index $(n=0.7$ and $n=1.5)$, in both diagrams increasing Reynolds number concludes higher pressure rise for the same time-mean flow rate therefore the definition of Reynolds indicates more pressure rise by increasing wave velocity, tube diameter and fluid density and also by decreasing power law index and fluid consistency.

Figure 5 shows the axial velocity profile in a fixed position for Newtonian and different non-Newtonian fluid. As it is shown all axial velocity profiles are parabolic form with zero tangent in the center line and the velocity reach to zero beside the wall. Increasing power law index results in higher velocity in the same radius of tube so there will be more flow rate for higher power law index fluid.

In Figures 6 and 7 the schematic of stream function ratio $\bar{\psi} / \bar{\psi}_{\text {Wall }}$ is depicted in half upper-section of flexible tube in the wave frame for two different kind of fluid. There is a region where the stream lines are closed and make the torus-shaped eddy in the hyperspace of the tube. This phenomenon is called trapping. On increasing power law index these eddies change in shape, eddies are longer and thinner in shear thinning case compared to shear thickening case. Also boluses become smaller by increasing flow rate through the tube in both case and they disappear when flow rate reaches to infinity which refers to Stokes flow in the rigid cosine form tube (non-peristaltic flow). Siddiqui and Schwars [14] show the trend of eddy disappearance for second order fluid by increasing flow rate. The existence of eddies effects on flow behavior and make a bit phase shift on upper stream functions, especially for higher flow rate. The affection of eddy existence on stream line bend decrease to zero for the lower stream line and completely depends on the form and size of eddies.

Figures 8 and 9 shows the perturbation parameter affection on stream function and eddies made by closed stream lines for $(\mathrm{n}<1)$. It is clear that more amplitude of wave or less tube diameter made bigger torus-shaped eddy and the smaller perturbation parameter make straighter stream line. In case (b) and (c) the central stream functions are not completely straight whereas in case (a) these stream lines are straight and also there is no eddy because of less amplitude ratio. The similar results are shown in Figure 9 for $(n>1)$. The comparison of Figure 8 and Figure 9 show that increasing power law index (n) make the bolus, shorter and wider and it is said before.

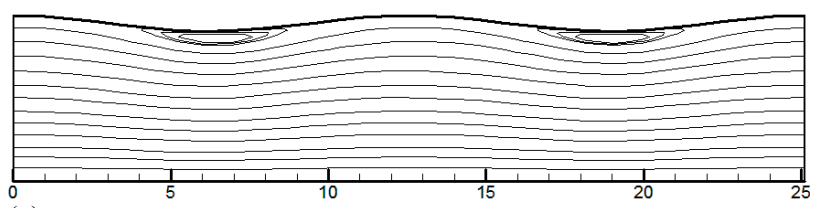

(a)

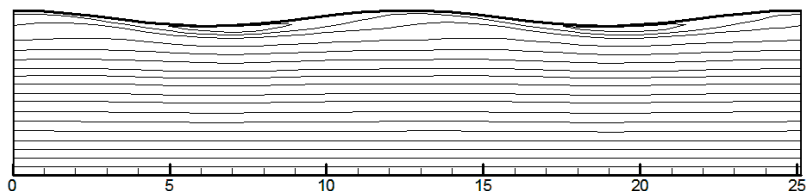

(b)

Figure 6: Schematic of stream function ratio $\bar{\psi} / \bar{\psi}_{\text {wall }}$ for $(n=0.7, \varepsilon=0.05, \alpha=0.5$, $\mathrm{Re}=50$ ) and (a) Lower flow rate; (b) Higher flow rate.

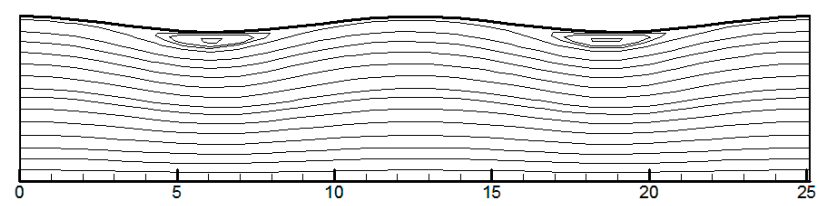

(a)

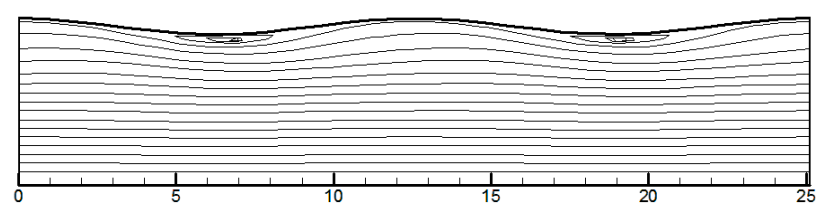

(b)

Figure 7: Schematic of stream function ratio $\bar{\psi} \bar{\psi}_{\text {Wall }}$ for $(n=1.5, \varepsilon=0.05, \alpha=0.5$, $\mathrm{Re}=50$ ) and (a) Lower flow rate; (b) Higher flow rate. 
Citation: Sadeghi K, Jalali Talab H (2014) Analytical Investigation of Peristaltic Transport of Power Law Fluid through a Tube. J Appl Mech Eng 3: 136. doi:10.4172/2168-9873.1000136

Page 6 of 6

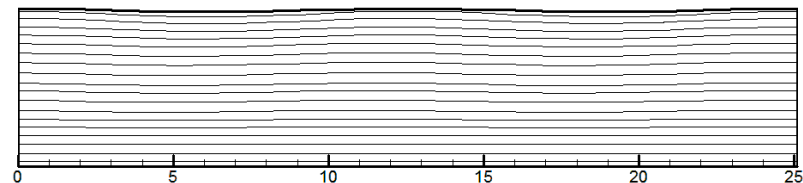

(a)

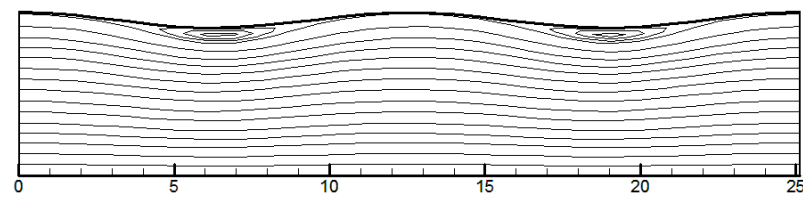

(b)

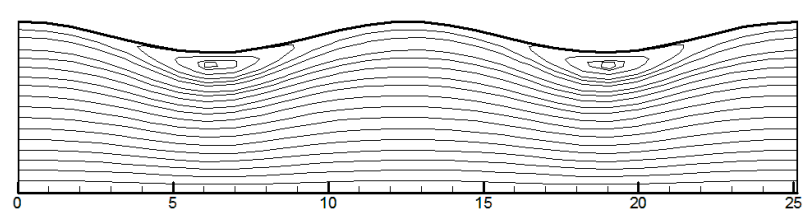

(c)

Figure 8: Schematic of stream function ratio $\bar{\psi} \bar{\psi}_{\text {wall }}$ for $(\mathrm{n}=0.7, \mathrm{\alpha}=0.5, \mathrm{Re}=50)$ and (a) $\varepsilon=0.01$; (b) $\varepsilon=0.05$; (c) $\varepsilon=0.1$.

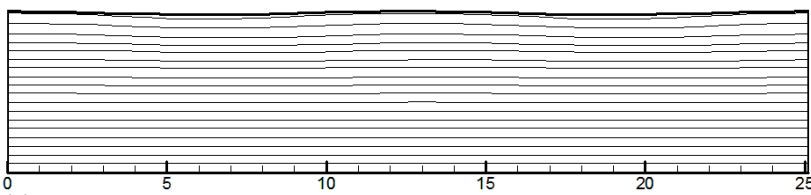

(a)

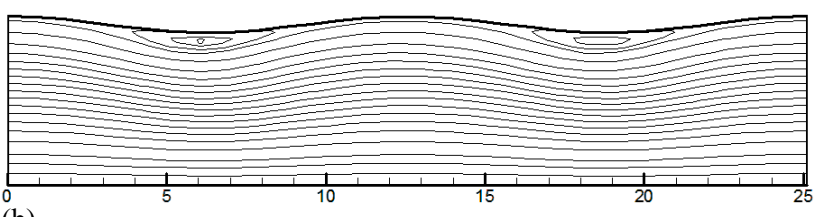

(b)

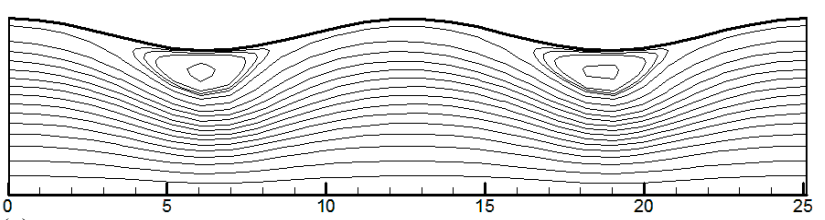

(c)

Figure 9: Schematic of stream function ratio $\bar{\psi} \bar{\psi}_{\text {Wall }}$ for $(\mathrm{n}=1.5, \mathrm{\alpha}=0.5, \mathrm{Re}=50)$ and (a) $\varepsilon=0.01$; (b) $\varepsilon=0.05$; (c) $\varepsilon=0.1$.

\section{Conclusion}

In this paper, the peristaltic flow of a power law fluid in a cylindrical channel is investigated by using a perturbation method in the wave frame. The effects of main parameters of flow rate, Reynolds number, flow behavior index $(n)$ and amplitude ratio $(\varepsilon)$ on the flow regime are studied. The results show that increment of power law index predicts more flow rate and less pressure rise in peristaltic motion. Trapping phenomenon is form by closed stream line depending on peristaltic motion.

\section{Acknowledgment}

Greatly appreciate S.M.A.Noori Rahim Abadi.

\section{References}

1. Latham TW (1966) Fluid Motion in a Peristaltic Pump. Massachusetts Thesis Massachusetts Institute of Technology, Cambridge, MA, USA

2. Shapiro AH (1967) Pumping and Retrograde Diffusion in Peristaltic Waves, In Proc Workshop Urethral Reflux in Children, Nat Acad Sci, Washington, DC.

3. Fung YC, Yih CS (1968) Peristaltic transport. JA \& Mech 85: 669-675

4. Radhakrishnamacharya $\mathrm{G}$ (1982) Long wave length approximation to peristaltic motion of power law fluid. Rheol Acta 21: 30-35.

5. Srivastava LM, Srivastava VP (1984) Peristaltic transport of blood: Casson model II. J Biomech 17: 821-829.

6. Mernone AV, Mazumdar JN (2002) A mathematical study of peristaltic transport of a Casson fluid. Mathematical and Computer Modeling 35: 895-912.

7. Srivastava LM, Srivastava VP (1988) Peristaltic transport of power-law fluid application to the ducts of efferent of the reproductive tract. Rheol Acta 27: 428-433.

8. Usha S, Rao AR (1997) Peristaltic transport of two layered Power-law fluids Trans ASME J Biomech Eng 199: 483-488.

9. Eytan O, Elad D (1999) Analysis of intra-uterine motion induced by uterine Contractions. Bull Math Biol 61: 221-238.

10. Eytan O, Jaffa AJ, Elad D (2001) Peristaltic flow in a tapered channel: application to embryo transport within the uterine cavity. Med Eng Phys 23: 473-482.

11. Mishra M, Rao AR (2003) Peristaltic transport of a Newtonian fluid in an Asymmetric channel. Z Angew Math Phys 54: 532-550.

12. Takabatake S, Ayukava K (1982) Numerical study of two-dimensional peristaltic flows. J Fluid Mech 122: 439-465.

13. Takabatake S, Ayukava K, Mori A (1988) Peristaltic pumping in circular cylindrical tubes: a numerical study of fluid transport and its efficiency. J Fluid Mech 193: 269-283.

14. Siddiqui AM, Schwars WH (1994) Peristaltic flow of a second-order fluid in tubes. J Non-Newtonian Fluid Mech 53: 257-284

15. Nadeem S, Akram S (2012) Influence of inclined magnetic field on peristaltic flow of a Jeffrey fluid with heat and mass transfer in an inclined symmetric or asymmetric channel. Asia Pacific Journal of Chemical Engineering 7: 33-44.

16. Nadeem S, Akram S (2012) Numerical and analytical solutions of peristaltic transport of a six constant Jeffreys model of Fluid in a symmetric or asymmetric channel. International Journal of fluid Mechanics Research 39: 238-260.

17. Akram S, Nadeem S (2012) Simulation of heat and mass transfer on peristaltic flow of hyperbolic tangent fluid in an asymmetric channel. International Journal for Numerical methods in fluids 70: 1475-1493.

18. Akram S, Ghafoor A, Nadeem S (2012) Mixed convective heat and Mass transfer on a peristaltic flow of a non-Newtonian fluid in a vertical asymmetric channel. Heat Transfer Asian Research 41: 613-633.

19. Akram S, Nadeem S (2013) Influence of induced magnetic field and heat transfer on the peristaltic motion of a Jeffrey fluid in an asymmetric channel: Closed form solutions. Journal of Magnetism and Magnetic Materials 328: 11 20.

20. Akram S, Nadeem S, Hanif M (2013) Numerical and analytical treatment on peristaltic flow of Williamson fluid in the occurrence of induced magnetic field Journal of Magnetism and Magnetic Materials 346: 142-151.

21. Nadeem S, Akram S, Akbar S, Noreen S (2013) Simulation of heat and chemical reactions on peristaltic flow of a Williamson fluid in an inclined asymmetric channel. Iranian journal of chemistry and chemical engineering 32: 93-107. 